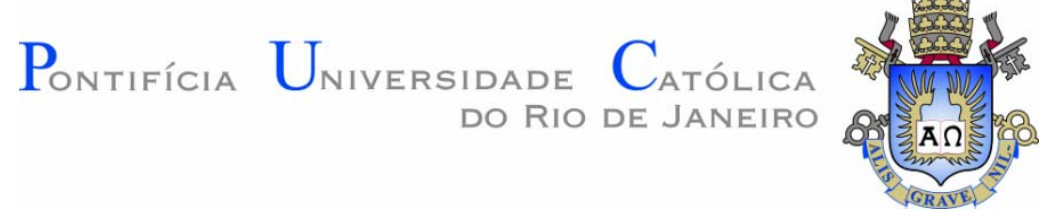

Claudia Ines Telleria Navarro

\title{
Caracterização Microestrutural das Fibras Naturais: Etlingera elatior, Costus e Helicônia bihai
}

Dissertação de Mestrado

Dissertação apresentada ao Programa de PósGraduação em Engenharia de Materiais e de Processos Químicos e Metalúrgicos da PUC-Rio como requisito parcial para obtenção do título de Mestre em Engenharia de Materiais e de Processos Químicos e Metalúrgicos

Orientador: Prof. Sidnei Paciornik Co-Orientador: Prof. José Roberto D’Almeida

Rio de Janeiro

Setembro de 2011 


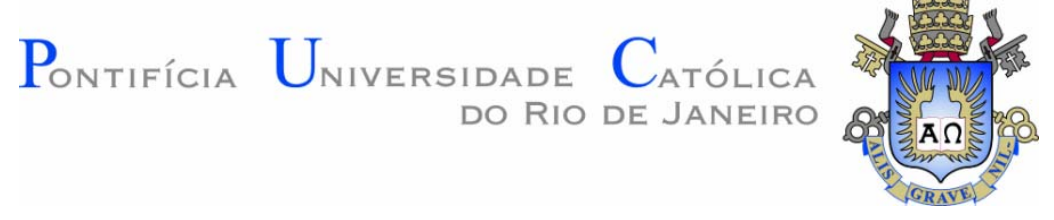

Claudia Ines Telleria Navarro

\title{
Caracterização Microestrutural das Fibras Naturais: Etlingera elatior, Costus, Helicônia bihai
}

\begin{abstract}
Dissertação apresentada como requisito parcial para obtenção do grau de Mestre pelo Programa de PósGraduação em Engenharia de Materiais e de Processos Químicos e Metalúrgicos do Departamento de Engenharia de Materiais do Centro Técnico Científico da PUC-Rio. Aprovada pela Comissão Examinadora abaixo assinada.
\end{abstract}

Prof. Sidnei Paciornik

Orientador

Departamento de Engenharia de Materiais - PUC-Rio

Prof. José Roberto Moraes d'Almeida

Co- Orientador

Departamento de Engenharia de Materiais - PUC-Rio

Prof. Marcos Henrique de Pinho Maurício Departamento de Engenharia de Materiais - PUC-Rio

Prof. $^{a}$ Ana Lucia Fampa Seabra d'Almeida UFRRJ

Prof. José Eugênio Leal Coordenador Setorial do Centro Técnico e Cientifico- PUC-Rio 
Todos os direitos reservados. É proibida a reprodução total ou parcial do trabalho sem autorização da universidade, da autora e do orientador.

\section{Claudia Ines Telleria Navarro}

Graduou-se em Engenharia Industrial na Universidade Autônoma Gabriel René Moreno, Santa Cruz - Bolívia, 2009. Áreas de interesse: Controle de Qualidade e Engenharia de Materiais.

Ficha Catalográfica

Navarro, Claudia Ines Telleria

Caracterização Microestrutural das Fibras Naturais: Etlingera elatior, Costus e Helicônia bihai / Claudia Ines Telleria Navarro; orientadores: Sidnei Paciornik; José Roberto D'Almeida . - 2011. 76 f. : il. (color.) ; $30 \mathrm{~cm}$

Dissertação (mestrado) - Pontifícia Universidade Católica do Rio de Janeiro, Departamento de Engenharia de Materiais, 2011. Inclui bibliografia

1. Engenharia de materiais - Teses. 2. Fibras naturais. 3. Análise de imagens. 4. Análise termogravimétrica. 5. Difração de raios-x. I. Paciornik Sidnei. II. D’Almeida, José Roberto Moraes. III. Pontifícia Universidade Católica do Rio de Janeiro. Departamento de Engenharia de Materiais. IV. Título.

CDD: 620.11 


\section{Agradecimentos}

A Deus pelas oportunidades.

Aos meus orientadores, Prof. Dr. Sidnei Paciornik e Prof. Dr. José Roberto Moraes d'Almeida pelo apoio e orientação.

Ao prof. Dr. Marcos Henrique de Pinho Maurício pela colaboração.

À Professora Verônica Calado da Escola de Química da UFRJ.

Aos professores e colaboradores do DEMa da PUC-Rio.

Aos meus pais pelo apoio constante.

Aos meus amigos.

Ao CAPES e PUC-Rio pelo suporte financeiro. 


\section{Resumo}

Navarro, Claudia Ines Telleria; Paciornik, Sidnei; d’Almeida, José Roberto Moraes. Caracterização Microestrutural das Fibras Naturais: Etlingera elatior, Costus, Helicônia bihai. Rio de Janeiro, 2011. 76p. Dissertação de Mestrado - Departamento de Engenharia de Materiais, Pontifícia Universidade Católica do Rio de Janeiro.

Fibras naturais vêm sendo utilizadas desde a antiguidade, mas, a partir do século XX, foram amplamente substituídas por fibras sintéticas, que apresentam diversas vantagens, tais como grande uniformidade de suas propriedades. Com a preocupação de se ter um desenvolvimento sustentável, voltou-se a substituir os materiais sintéticos por materiais naturais. Algumas das vantagens de utilizar fibras ligno-celulósicas incluem biodegrabilidade, a fixação de carbono, baixo custo e baixa abrasividade. Nos últimos anos, entre diversas aplicações, os setores de construção civil e automobilística estão utilizando fibras naturais como reforço de compósitos termoplásticos, assim como para interiores de cabines de automóveis e caminhões e em capacetes usados em EPI. Este trabalho visa realizar uma caracterização de fibras naturais existentes no mercado brasileiro, a saber: Bastão do Imperador, Costus e Helicônia bihai. O principal uso dessas três espécies se dá como flores ornamentais e como plantas medicinais, mas há um grande potencial no reaproveitamento dos rejeitos dos caules dessas plantas. Para isto foi realizada uma caracterização das fibras, visando medir a verdadeira seção transversal destas utilizando microscopia digital e análise de imagens. Alem disto foi desenvolvida uma metodologia própria para a preparação de amostras de fibras naturais. Foi realizado caracterização por MEV, DRX e TGA.

\section{Palavras-Chave}

Fibras naturais; Análise de Imagens; Análise Termogravimétrica; Difração de Raios-X. 


\section{Abstract}

Navarro, Claudia Ines Telleria; Paciornik, Sidnei (Advisor); d'Almeida, José Roberto Moraes (co-advisor). Microestructural Characterization of Natural Fibers: Etlingera elatior, Costus, Heliconia bihai. Rio de Janeiro, 2011. 76p. MSc. Dissertation - Departamento de Engenharia de Materiais, Pontifícia Universidade Católica do Rio de Janeiro.

Natural fibers have been used since ancient times, but since the 20th century they have been largely substituted by synthetic fibers, which offer several advantages such as their homogeneous properties. However, in light of the growing concerns about sustainable development, natural materials have once again started to substitute synthetic ones. A few of the advantages of utilizing lignocellulosic fibers include biodegradability, low cost, and low abrasiveness. In recent years, among many applications, the civil construction and automotive industries are using natural fibers as reinforcement in thermoplastic composites, as well as interior cabins of cars and trucks and helmets used in PPE. Over the past years, areas such as civil construction and the automotive industry have used natural fiber-reinforced thermoplastic composites in car and truck interiors, as well as in helmets used in constructions. This work intends to characterize fibers available in the brazilian market, in particular: Etlingera elatior, Costus, Helicônia bihai. These three species are mainly used as medicinal plants or ornamental flowers, but there is a great potential to reutilize the discarded stems of these plants. Furthermore, these fibers will be characterized using digital microscopy and image analysis, aiming to measure their real transversal section. A method for preparing natural fiber samples will be developed for this purpose. For this was a characterization of the fibers was done in order to measure the true cross section of these using digital microscopy and image analysis. Besides it has been developed a methodology for the preparation of samples of natural fibers. Characterization was performed by SEM, XRD and TGA.

\section{Keywords}

Natural fibers; Image Analyses; Thermogravimetric Analysis; X-ray diffraction. 


\section{Sumário}

1 Introdução 13

$\begin{array}{ll}\text { 1.1. Objetivo } & 15\end{array}$

2 Revisão Bibliográfica $\quad 16$

2.1. Fibras Ligno-celulósicas 16

2.1.1. Celulose 17

2.1.2. Hemicelulose 18

2.1.3. Lignina 19

2.2. Microscopia Eletrônica de Varredura 21

2.3. Análise da seção transversal 25

2.4. Difração de Raios-X 27

$\begin{array}{ll}\text { 2.5. Termogravimetria } & 29\end{array}$

3 Materiais e Métodos $\quad 35$

3.1. Fibras utilizadas $\quad 35$

3.2. Família Zingiberaceae $\quad 37$

3.2.1. Bastão do Imperador 38

3.2.2. Costus 39

3.2.3. Helicônia 40

3.3. Preparação das amostras $\quad 42$

3.3.1. Preparação das amostras para analise no Microscópio 42

3.3.2. Preparação das amostras para Difração de Raios-X e análise Termogravimétrica 43

3.4. Microscopia Eletrônica de Varredura (MEV) 43

3.5. Difração de Raios-X 44

3.6. Analise Termogravimétrica (TGA) 44

3.7. Análise da Seção Transversal das fibras ligno-celulósicas 45

$\begin{array}{ll}\text { 3.7.1. Parâmetros de tamanho } & 47\end{array}$

$\begin{array}{ll}\text { 3.7.2. Inclinação das fibras e fibras partidas } & 47\end{array}$ 
4 Resultados e Discussão $\quad 49$

4.1. Microscopia Eletrônica de Varredura 49

4.2. Difração de Raios-X 54

4.3. Termogravimetria 58

4.4. Medição da seção transversal 62

5 Conclusões $\quad 70$

6 Referências bibliográficas $\quad 72$ 


\section{Lista de Figuras}

Figura 1 - Estrutura da celulose, esquema da ilustração das moléculas de celulose [7]

Figura 2 - Estrutura física da fibra ligno-celulósica [9].

Figura 3 - Posições onde ocorre à formação de pontes de hidrogênio intermoleculares [7].

Figura 4 - Ligações Polioses (Hemiceluloses) com Celulose (micro fibrilas) [7].

Figura 5 - Algumas fórmulas dos açucares componentes das Hemiceluloses [7].

Figura 6 - Estrutura Geral da lignina [13].

Figura 7 - Unidades presentes na lignina: (1) p-hidroxifenila,

(2) guaiacila, (3) siringila [6].

Figura 8 - Superfícies da fibra de coco (a) vista longitudinal,

(b) vista frontal [6].

22

Figura 9 - Micrografias das fibras de algodão (a) e de sisal (b) [16].

Figura 10 - Micrografias das fibras (a) açaí, (b) coco e

(c) juta mostrando as seções longitudinais [17].

Figura 11 - Micrografias da seção transversal das fibras (a) açaí,

(b) coco e (c) juta, com a medida dos seus respectivos diâmetros [17].

Figura 12 - Micrografia óptica da seção transversal em um compósito reforçado com fibra de curauá e a aproximação para a forma de um polígono [18].

Figura 13 - Mosaicos de fibras de (a) piaçava, (b) juta e

(c) pupunha [2].

Figura 14 - Difratograma da fibra de Tururi [26]. 28

Figura 15 - Difratograma da pupunha [22].

Figura 16 - Gráfico esquemático de TGA (vermelho) e sua derivada (azul) [27].

Figura 17 - Curvas de TGA (a) e DTG (b) para Hemicelulose, Celulose 
e Lignina [28].

Figura 18 - Curvas TGA/ DTG da fibra de sisal, atmosfera de nitrogênio, taxa de aquecimento de $10^{\circ} \mathrm{C} / \mathrm{Min}$ [21].

Figura 19 - Curva de TGA da fibra de cana-de-açúcar e sua derivada TGA [29].

Figura 20 - (a) curva de TGA, (b) curva de DTG para piaçava sem tratar e tratadas (mercerizadas). Estudo comparativo [30].

Figura 21 - Curva de TGA e curva de DTG, para pupunha [22].

Figura 22 - Localização do Município de Rio Bonito, no mapa do Brasil (a) e do Rio de Janeiro (b) [31].

Figura 23 - Moenda de cana de açúcar, utilizada para processamento das fibras de Bastão do Imperador, Costus e Helicônia [32].

Figura 24 - Principais regiões onde se cultiva Bastão do Imperador, Helicônia e Costus [33].

Figura 25 - Família Zingiberaceae [36].

Figura 26 - Inflorescência da planta Bastão do Imperador [37]. 39

Figura 27 - Inflorescência da planta Costus [40].

Figura 28 - Inflorescência da planta Helicônia [41].

Figura 29 - Procedimento para preparação da seção transversal das fibras: (a) imersão das fibras em água, (b) corte das fibras.

Figura 30 - Amostra de Helicônia, fibras cortadas para as análises de Raios-X e TGA.

Figura 31 - (a) Equipamento de análise termogravimétrica e

(b) cadinho de platina.

Figura 32 - Medição manual da seção transversal da fibra Costus. 46

Figura 33 - Lista de recursos oferecidos pelo programa AxioVision. 46

Figura 34 - Parâmetros de tamanho [].

Figura 35 - (a) Fibra de Helicônia com problema de inclinação,

(b) fibra de Costus partida.

Figura 36 - Micrografia obtida por MEV da fibra de Bastão do Imperador, (a) superfície longitudinal e (b) seção transversal da fibra.

Figura 37- Micrografia obtida por MEV da fibra de Costus,

(a) superfície longitudinal e (b) seção transversal da fibra. 
Figura 38 - Micrografia obtida por MEV da fibra de Helicônia,

(a) superfície longitudinal e (b) seção transversal da fibra.

Figura 39 - Micrografia obtida por MEV detalhando as microfibrilas da fibra de Bastão do Imperador.

Figura 40 - Micrografia obtida por MEV detalhando os vasos

condutores das microfibrilas da fibra de Costus.

Figura 41 - Micrografia obtida por MEV detalhando os vasos condutores das micro fibrilas da fibra de Helicônia.

Figura 42 - Difratograma da Fibra de Bastão do Imperador.

55

Figura 43 - Difratograma da Fibra de Costus (Difração de Raios-X). 55

Figura 44 - Difratograma da Fibra de Helicônia (difração de Raios-X). 56

Figura 45 - Curvas de TGA (vermelho) e DTG (azul) para as fibras

de Bastão do Imperador.

58

Figura 46 - Curvas de TGA (vermelho) e DTG (azul) da fibra de

Costus.

Figura 47 - Curva de TGA (vermelho) e DTG (azul) da fibra de Helicônia.

60

Figura 48 - DTG para Pectina em pó [44].

61

Figura 49 - Distribuição de diâmetros das fibras Bastão do Imperador. 62

Figura 50 - Distribuição de diâmetros das fibras de Costus.

63

Figura 51 - Distribuição de diâmetros das fibras de Helicônia.

Figura 52 - Caixa e bigode dos diâmetros das fibras Costus, Bastão do Imperador e Helicônia.

Figura 53 - Histograma de Razão de Aspectos para as fibras Bastão do Imperador.

Figura 54 - Histograma de Razão de Aspectos para as fibras de Costus.

Figura 55 - Histograma de razão de aspectos para as fibras de Helicônia. 


\section{Lista de Tabelas}

Tabela 1 - Valores estatísticos $\Delta$ Fmax e $\Delta$ Fmin para as fibras de piaçava, juta e pupunha. 26

Tabela 2 - Picos nos difratogramas difração de Raios-X. 56

Tabela 3 - Índice de Cristalinidade. $\quad 56$

Tabela 4 - Picos nos difratogramas difração de Raios-X (comparativo) 56

Tabela 5 - Perda de umidade para as três fibras na primeira etapa. $\quad 59$

Tabela 6 - Comparação de temperaturas de degradação

(TGA-DTG). $\quad 60$

Tabela 7 - Diferença significativa entre diâmetros médios. $\quad 66$

Tabela 8 - Diferença significativa entre Razão de Aspectos. 66

Tabela 9 - Parâmetros calculados da fibra Helicônia. 67

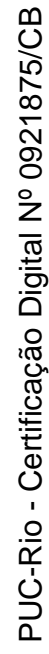

Tabela $10-\Delta$ Fmax e $\Delta$ Fmin para os três tipos de fibras medidas. $\quad 68$ 